\title{
KamLAND results and the radiogenic terrestrial heat
}

\author{
Gianni Fiorentini, $1,2, *$ Marcello Lissia, ${ }^{3,4, \dagger}$ Fabio Mantovani, $, 5,6,2$, , and Barbara Ricci ${ }^{1,2}$, \& \\ ${ }^{1}$ Dipartimento di Fisica, Università di Ferrara, I-44100 Ferrara, Italy \\ ${ }^{2}$ Istituto Nazionale di Fisica Nucleare, \\ Sezione di Ferrara, I-44100 Ferrara, Italy \\ ${ }^{3}$ Istituto Nazionale di Fisica Nucleare, \\ Sezione di Cagliari, I-09042 Monserrato (CA), Italy \\ ${ }_{4}^{4}$ Dipartimento di Fisica, Università di Cagliari, I-09042 Monserrato (CA), Italy \\ ${ }^{5}$ Dipartimento di Scienze della Terra, \\ Università di Siena, I-53100 Siena, Italy \\ ${ }^{6}$ Centro di GeoTecnologie CGT, I-52027 San Giovanni Valdarno, Italy
}

(Dated: September 2, 2005)

\begin{abstract}
We find that recent results from the KamLAND collaboration on geologically produced antineutrinos, $\mathrm{N}(\mathrm{U}+\mathrm{Th})=28_{-15}^{+16}$ events, correspond to a radiogenic heat production from Uranium and Thorium decay chains $\mathrm{H}(\mathrm{U}+\mathrm{Th})=38_{-33}^{+35} \mathrm{TW}$. The $99 \%$ confidence limit on the geo-neutrino signal translates into the upper bound $\mathrm{H}(\mathrm{U}+\mathrm{Th})<162 \mathrm{TW}$, which is much weaker than that claimed by KamLAND, $\mathrm{H}(\mathrm{U}+\mathrm{Th})<60 \mathrm{TW}$, based on a too narrow class of geological models. We also performed an analysis of KamLAND data including recent high precision measurements of the ${ }^{13} \mathrm{C}(\alpha, n){ }^{16} \mathrm{O}$ cross section. The result, $\mathrm{N}(\mathrm{U}+\mathrm{Th})=31_{-13}^{+14}$, corroborates the evidence $(\simeq 2.5 \sigma)$ for geo-neutrinos in KamLAND data.
\end{abstract}

PACS numbers: 91.35.-x, 13.15.+g, 14.60.Pq, 23.40.Bw

\footnotetext{
*Electronic address: fiorenti@fe.infn.it

${ }^{\dagger}$ Electronic address: marcello.lissia@ca.infn.it

${ }^{\ddagger}$ Electronic address: mantovani@fe.infn.it

${ }^{\S}$ Electronic address: ricci@fe.infn.it
} 


\section{INTRODUCTION}

Geologically produced antineutrinos (geo-neutrinos) were introduced by Eder [1] in the sixties and Marx 2] soon realized their relevance. In the eighties Krauss et al. discussed their potential as probes of the Earth's interior in an extensive publication [3]. In the nineties the first paper on a geophysical journal was published by Kobayashi et al. [4]. In 1998, Raghavan et al. [5] and Rotschild et al. 6] pointed out that KamLAND and Borexino should be capable of geo-neutrino detection.

The potential of geo-neutrinos for providing information on the energetics and composition of the Earth has been discussed in Refs. 7, 8] and more recently in Ref. [9]. The KamLAND collaboration has just published [10] their experimental results, claiming some 28 geo-neutrino events from Uranium and Thorium decay chains in a two-year exposure. This important step shows that the technique for exploiting geo-neutrinos in the investigation of the Earth's interior is now available. In order to understand where to go with geo-neutrinos, we have to know where we stand, in the light of the available data. In this spirit, the aim of this letter is to discuss the implication of the KamLAND result on the contribution of Uranium and Thorium decay chains to the terrestrial heat.

\section{THE GEO-NEUTRINO SIGNAL AND THE RADIOGENIC TERRESTRIAL HEAT}

For a given value of, e.g., the Uranium mass in the Earth, $m(\mathrm{U})$, the contributed heat production rate from the Uranium decay chain is uniquely determined, $\mathrm{H}(\mathrm{U})=0.95 \mathrm{TW}$ $\times m(\mathrm{U}) /\left(10^{16} \mathrm{~kg}\right)$, whereas the flux and signal of geo-neutrinos depend on the detector location and on the Uranium distribution inside the Earth. The connection between the signal of geo-neutrinos from the Uranium decay chain, the mass of Uranium in the Earth and the heat production rate from that element was found in Ref. [9], by using global mass balance together with a detailed geochemical and geophysical study of the region surrounding Kamioka.

We remark that the mass of Uranium in the crust, $m_{c}(\mathrm{U})$, is rather well constrained by geological data, in the interval $(3 \div 4) \times 10^{16} \mathrm{~kg}$. The main uncertainty is the amount of Uranium (and Thorium) in the mantle. Geo-neutrinos should provide us with this informa- 
tion.

For a total uranium mass $m(\mathrm{U})$ in the Earth, the maximal and minimal signals can be derived by using a proximity argument: the maximal (minimal) signal is obtained by placing the sources as close (as far) as possible to (from) the detector, consistently with geochemical and geophysical constraints. The maximal signal is thus obtained for an Uranium rich crust, $m_{c}(\mathrm{U})=4 \times 10^{16} \mathrm{~kg}$, and distributing uniformly in the mantle the rest of Uranium, $m(\mathrm{U})-m_{c}(\mathrm{U})$. The minimal signal corresponds to an Uranium poor crust, $m_{c}(\mathrm{U})=3 \times 10^{16}$ $\mathrm{kg}$, and distributing the rest near the bottom of the lower mantle. These maximal and minimal signals provide the borders of Fig. 5 of Ref. [9], where the interested reader can find more details.

We have extended the same calculations to Thorium, assuming a global chondritic Uranium-to-Thorium mass ratio, $m(\mathrm{Th}) / m(\mathrm{U})=3.9 \pm 0.1$, so that we can now connect the combined signal of geo-neutrinos from Uranium and Thorium progenies, $\mathrm{S}(\mathrm{U}+\mathrm{Th})$, with the radiogenic heat production rate from these elements, $\mathrm{H}(\mathrm{U}+\mathrm{Th})$, see Fig. 1.

The geo-neutrino signal is expressed in Terrestrial Neutrino Units, one TNU corresponding to $10^{-32} \overline{\nu_{e}}$ captures per target proton per year.

The allowed band in Fig. 1 is estimated by considering rather extreme ${ }^{1}$ models for the distributions of radioactive elements, chosen so as to maximize or minimize the signal for a given heat production rate [9].

We also remark that, in comparison with the experimental error,the width of the band is so narrow that we can limit the discussion to the median line of the allowed band in Fig. 11, which represents our best estimate for the relationship between signal and power.

For the Bulk Silicate Earth (BSE) model, $\mathrm{H}(\mathrm{U}+\mathrm{Th})=16 \mathrm{TW}$, our prediction for Kamioka is centered at $37 \mathrm{TNU}$.

By assuming that Uranium and Potassium in the Earth are in the ratio $1 / 10,000$ and that there is no Potassium in the core, the total radiogenic power is $\mathrm{H}(\mathrm{U}+\mathrm{Th}+\mathrm{K})=1.18$ $\mathrm{H}(\mathrm{U}+\mathrm{Th})$. With these assumptions, a maximal and fully radiogenic heat production rate, $\mathrm{H}(\mathrm{U}+\mathrm{Th}+\mathrm{K})=44 \mathrm{TW}$, corresponds to $\mathrm{H}(\mathrm{U}+\mathrm{Th})=37 \mathrm{TW}$, which gives a signal $\mathrm{S}(\mathrm{U}+\mathrm{Th})$ $\approx 56 \mathrm{TNU}$.

\footnotetext{
${ }^{1}$ We are neglecting here the possibility that some Uranium or Thorium is hidden in the core. This possibility, which has been advanced by some authors (see, e.g., Herndon 11] and Hofmeister and Criss [12]) would imply an even smaller signal-to-power ratio.
} 
The KamLAND collaboration has reported [10] data from an exposure of $\mathrm{N}_{p}=(0.346 \pm$ $0.017) \times 10^{32}$ free protons over a time $T=749$ days with a detection efficiency $\epsilon=69 \%$ : the effective exposure is thus $\mathrm{E}_{\text {eff }}=\mathrm{N}_{p} \times T \times \epsilon=(0.487 \pm 0.025) \times 10^{32}$ protons $\cdot$ yr. In the energy region where geo-neutrinos are expected, there are $\mathrm{C}=152$ counts, implying a statistical fluctuation of \pm 12.5 . Of these counts, a number $R=80.4 \pm 7.2$ are attributed to reactor events, based on an independent analysis of higher energy data. Fake geo-neutrino events, originating from ${ }^{13} \mathrm{C}(\alpha, n){ }^{16} \mathrm{O}$ reactions following the alpha decay of contaminant ${ }^{210} \mathrm{Po}$, are estimated to be $\mathrm{F}=42 \pm 11$, where the error is due to a $20 \%$ uncertainty on the ${ }^{13} \mathrm{C}(\alpha, \mathrm{n}){ }^{16} \mathrm{O}$ cross section and a $14 \%$ uncertainty on the number of ${ }^{210} \mathrm{Po}$ decays in the detector. Other minor backgrounds account for $\mathrm{B}=4.6 \pm 0.2$ events. The number of geoneutrino events is estimated by subtraction, $\mathrm{N}(\mathrm{U}+\mathrm{Th})=\mathrm{C}-\mathrm{R}-\mathrm{F}-\mathrm{B}$, with an uncertainty obtained by combining the independent errors: $\mathrm{N}(\mathrm{U}+\mathrm{Th})=25_{-18}^{+19}$. The geo-neutrino signal is thus $\mathrm{S}(\mathrm{U}+\mathrm{Th})=\mathrm{N}(\mathrm{U}+\mathrm{Th}) / \mathrm{E}_{\text {eff }}=51_{-36}^{+39} \mathrm{TNU}$. From the median line in Fig. 1 one finds

$$
\mathrm{H}(\mathrm{U}+\mathrm{Th})=31_{-31}^{+43} \mathrm{TW} \quad \text { (rate only) }
$$

This "rate only" study has been improved in Ref. [10] by exploiting the shape of the spectrum. A likelihood analysis of the unbinned spectrum yields $\mathrm{N}(\mathrm{U}+\mathrm{Th})=28_{-15}^{+16}$, see Fig. $4 \mathrm{~b}$ of Ref. [10]. This implies $\mathrm{S}(\mathrm{U}+\mathrm{Th})=57_{-31}^{+33} \mathrm{TNU}$ and

$$
\mathrm{H}(\mathrm{U}+\mathrm{Th})=38_{-33}^{+35} \mathrm{TW} \quad \text { (rate }+ \text { spectrum) }
$$

The best fit value is close to the maximal and fully radiogenic model, however the BSE is within $1 \sigma$.

By using the median line in Fig. 1, the 99\% confidence limit on the signal (145 TNU) corresponds to $133 \mathrm{TW}$. If we include the uncertainty band of the theoretical models, we find an upper bound of $162 \mathrm{TW}$, see point A in Fig. 1. This point corresponds to a model with a total Uranim mass $m(\mathrm{U})=80 \times 10^{16} \mathrm{~kg}$, an uranium poor crust, $m_{c}(\mathrm{U})=3 \times 10^{16}$ $\mathrm{kg}$, the rest of the Uranium being placed at the bottom of the mantle, and global chondritic Thorium-to-Uranium ratio.

This 162 TW upper bound is much higher than the 60 TW upper bound claimed in Ref. [10], which was obtained by using a family of geological models which are too narrow and are also incompatible with well-known geochemical and geophysical data. 
In fact, the authors of Ref. [10] start with a reference BSE model derived from Ref. [13]: the total Uranium mass is $m_{\mathrm{BSE}}=8 \times 10^{16} \mathrm{~kg}$, roughly half in the crust and the rest in the mantle, and the abundance ratio is $\mathrm{Th} / \mathrm{U}=3.9$. This model corresponds to $\mathrm{H}_{\mathrm{BSE}}(\mathrm{U}+\mathrm{Th})=$ $16 \mathrm{TW}$ and predicts a signal of $38.5 \mathrm{TNU}$, very close to our prediction for BSE. The signal is assumed to scale with the total mass of $\mathrm{U}+\mathrm{Th}$, so that heat production and signal are also proportional:

$$
\mathrm{S}(\mathrm{U}+\mathrm{Th})=38.5 \mathrm{TNU} \times \mathrm{H}(\mathrm{U}+\mathrm{Th}) /(16 \mathrm{TW}) .
$$

In this way, the $99 \%$ upper limit on the signal, $145 \mathrm{TNU}$, is translated into $60 \mathrm{TW}$ [10], see point B in Fig. 1.

This scaling assumption, however, produces a too limited series of models. The points in the shaded area of Fig. 1] correspond to all models which are compatible with available geochemical and geophysical data ${ }^{2}$ : most of these models cannot be obtained by Eq. 3 and predict, for a given signal, a larger power than Eq. 3. which therefore cannot be used to derive an upper bound on the radiogenic power production.

Furthermore, Eq. 3 implies that Uranium in the crust (and in the mantle) scales linearly with the total Uranium mass. This becomes incompatible with the geochemical data on the crust $\left(m_{c}(\mathrm{U})<4 \times 10^{16} \mathrm{~kg}\right)$ already for total masses slightly above the BSE estimate, i.e., for models where $\mathrm{H}(\mathrm{U}+\mathrm{Th})>20 \mathrm{TW}$. For example, in the model yielding $60 \mathrm{TW}$ (point B in Fig. 1) the crust should contains about $13 \times 10^{16} \mathrm{~kg}$ of Uranium, four times more than the largest geochemical estimate. This inconsistency is clearly seen in Fig. 1, which shows that the family of models labeled as "rescaled models" lies essentially in the geo-chemically excluded region.

We remark that the bound $\mathrm{H}(\mathrm{U}+\mathrm{Th})<162 \mathrm{TW}$ which we have extracted from KamLAND data does not add any significant information on Earth's interior, since anything exceeding $\mathrm{H}(\mathrm{U}+\mathrm{Th})=37 \mathrm{TW}$ (i.e. $\mathrm{H}(\mathrm{U}+\mathrm{Th}+\mathrm{K})=44 \mathrm{TW})$ is unrealistic. The upper limit simply reflects the large uncertainty in this pioneering experiment.

On the other hand, what is important for deciding the potential of future experiments is the relationship between geo-neutrino signal and heat production in the physically interesting region, $\mathrm{H}(\mathrm{U}+\mathrm{Th}) \leq 37 \mathrm{TW}$. The basic parameter is the slope, $\mathrm{dS} / \mathrm{dH}$, which expresses how

\footnotetext{
${ }^{2}$ We note that actually models with $\mathrm{H}(\mathrm{U}+\mathrm{Th})>37 \mathrm{TW}$ are essentially unrealistic; this portion of the graph is included just for discussing KamLAND results.
} 
the experimental error translates into an uncertainty on the deduced heat production. For our models we find from Fig. 1 dS/dH $\simeq 1 \mathrm{TNU} / \mathrm{TW}$. Discrimination between BSE and fully radiogenic models, which requires a precision $\Delta \mathrm{H} \sim 7 \mathrm{TW}$, requires thus an experiment with an accuracy $\Delta \mathrm{S} \sim 7 \mathrm{TNU}$. The "rescaled models" of ref. [10], yielding $\mathrm{dS} / \mathrm{dH} \simeq 2.4$ TNU/TW, might misleadingly suggest that the same goal can be achieved for $\Delta \mathrm{S}=17 \mathrm{TNU}$.

\section{THE GEO-NEUTRINO SIGNAL AND THE $\left.{ }^{13} \mathbf{C}(\alpha, n)\right)^{16}$ O CROSS SECTION.}

As already remarked, a major uncertainty for extracting the geo-neutrino signal originates from the ${ }^{13} \mathrm{C}(\alpha, n){ }^{16} \mathrm{O}$ cross section ${ }^{3}$. The values used in Ref. 10] are taken from the JENDL 14] compilation, which provides an R-matrix fit of relatively old data. A 20\% overall uncertainty has been adopted in [10], corresponding to the accuracy claimed in the original experimental papers (see, e.g., Ref. [17]).

Recently a series of high precision measurements for this cross section has been performed 15]. In the relevant energy range $(1 \div 5.3) \mathrm{MeV}$, the absolute normalization has been determined within a $4 \%$ accuracy. The measured values are generally in very good agreement with those recommended in JENDL, see Fig. 2, however, we find that the neutron yield per alpha particle is $5 \%$ smaller. It follows that the number of fake neutrinos is lower, $\mathrm{F}=$ $40 \pm 5.8$, and geo-neutrino events obviously increase ${ }^{4}$.

The "rate only" analysis gives now $27_{-15}^{+16}$ geo-neutrino events, corresponding to $\mathrm{S}(\mathrm{U}+\mathrm{Th})$ $=55_{-31}^{+33}$ TNU. From the median line of Fig. 1 the radiogenic power is now:

$$
\mathrm{H}(\mathrm{U}+\mathrm{Th})=36_{-33}^{+35} \mathrm{TW} \quad\left(\text { rate }+ \text { new }{ }^{13} \mathrm{C}(\alpha, n){ }^{16} \mathrm{O}\right)
$$

We also performed an analysis ${ }^{5}$ of the binned spectrum reported in Fig. 3 of Ref. [10]. This analysis gives $\mathrm{N}(\mathrm{U}+\mathrm{Th})=31_{-13}^{+14}$ counts, corresponding to $\mathrm{S}(\mathrm{U}+\mathrm{Th})=63_{-25}^{+28} \mathrm{TNU}$ and

\footnotetext{
${ }^{3}$ In fact, the claim of 9 geo-neutrino events in Ref. 16] should be dismissed: more than half of these events are to be considered as fake signal, produced from ${ }^{13} \mathrm{C}(\alpha, n){ }^{16} \mathrm{O}$ reaction.

${ }^{4}$ Indeed Ref. 10] mentions that an alternative analysis including the time structure of the scintillation light from different particles produced a slightly larger geo-neutrino signal, which is consistent with the result presented here.

${ }^{5}$ A complete analysis requires several details (the un-binned spectrum, the energy dependence of the detection efficiency, ...) which are not available to us. Just for a comparison, the binned spectrum analysis using the JENDL cross sections with $20 \%$ uncertainty gives us $\mathrm{N}(\mathrm{U}+\mathrm{Th})=28.5_{-14}^{+15}$, in agreement with [10].
} 
thus:

$$
\mathrm{H}(\mathrm{U}+\mathrm{Th})=44_{-27}^{+31} \mathrm{TW} \quad\left(\text { rate }+ \text { spectrum }+ \text { new }{ }^{13} \mathrm{C}(\alpha, \mathrm{n}){ }^{16} \mathrm{O}\right)
$$

\section{CONCLUDING REMARKS}

In summary, the new data on ${ }^{13} \mathrm{C}(\alpha, n){ }^{16} \mathrm{O}$ corroborate the evidence for geo-neutrinos in KamLAND data, which becomes near to $2.5 \sigma$.

On the other hand, the determination of radiogenic heat power from geo-neutrino measurements is still affected by a $70 \%$ uncertainty. The best fit of $\mathrm{H}(\mathrm{U}+\mathrm{Th})$ is close to the prediction of a maximal and fully radiogenic model, however the BSE prediction is within $1 \sigma$ from it.

With more statistics KamLAND should be capable of providing a three sigma evidence of geo-neutrinos, but discrimination between BSE and fully radiogenic models definitely requires new detectors, with class and size similar to that of KamLAND, far away from nuclear power plants.

\section{Acknowledgments}

We are grateful to C. Rolfs and his group for useful discussions and for allowing us to use their results.

We thank for their useful comments A. Bottino, E. Lisi, W. F. McDonough, and R. Raghavan. We appreciated the suggestions of the anonymous referee.

[1] G. Eder, Nucl. Phys. 78 (1966) 657.

[2] G. Marx, Czech. J. Phys B 19 (1969) 1471.

[3] L.M. Krauss et al., Nature 310 (1984) 191.

[4] M. Kobayashi and Y. Fukao, Geophy. Res. Lett. 18 (1991) 633.

[5] R.S. Raghavan et al., Phys. Rev. Lett. 80 (1998) 635.

[6] G.C. Rotschild et al., Geophy. Res. Lett. 25 (1998) 1083.

[7] G. Fiorentini, F. Mantovani and B. Ricci, Phys. Lett. B 557 (2003) 139 arXiv:nucl-ex/0212008. 
[8] F. Mantovani, L. Carmignani, G. Fiorentini and M. Lissia, Phys. Rev. D 69 (2004) 013001 arXiv:hep-ph/0309013.

[9] G. Fiorentini, M. Lissia, F. Mantovani and R. Vannucci, Phys. Rev. D 72 (2005) 033017 arXiv:hep-ph/0501111.

[10] T. Araki et al (KamLAND coll.), Nature 436 (2005) 499.

[11] J. M. Herndon, Proc. Natl. Acad. Sci. USA 93 (1996) 646-648, Geophysics.

[12] A.M. Hofmeister and R.E. Criss, Tectonophysics 395 (2005) 195.

[13] S. Enomoto, Thesis, Tohoku Univ. (2005), http://www.awa.tohoku.jp/KamLAND/publications/Sanshiro_thesis.pdf.

[14] JENDL Japanese Evaluated Nuclear Data Library, http://wwwndc.tokai.jaeri.go.jp/jendl/

[15] S. Harissopulos et al., Phys. Rev. C (2005) to appear arXiv:nucl-ex/0509014.

[16] K. Eguchi et al. [KamLAND Collaboration], Phys. Rev. Lett. 90 (2003) 021802 arXiv:hep-ex/0212021.

[17] J.K. Bair and F.X. Haas Phys. Rev. C 7 (1973) 1356. 


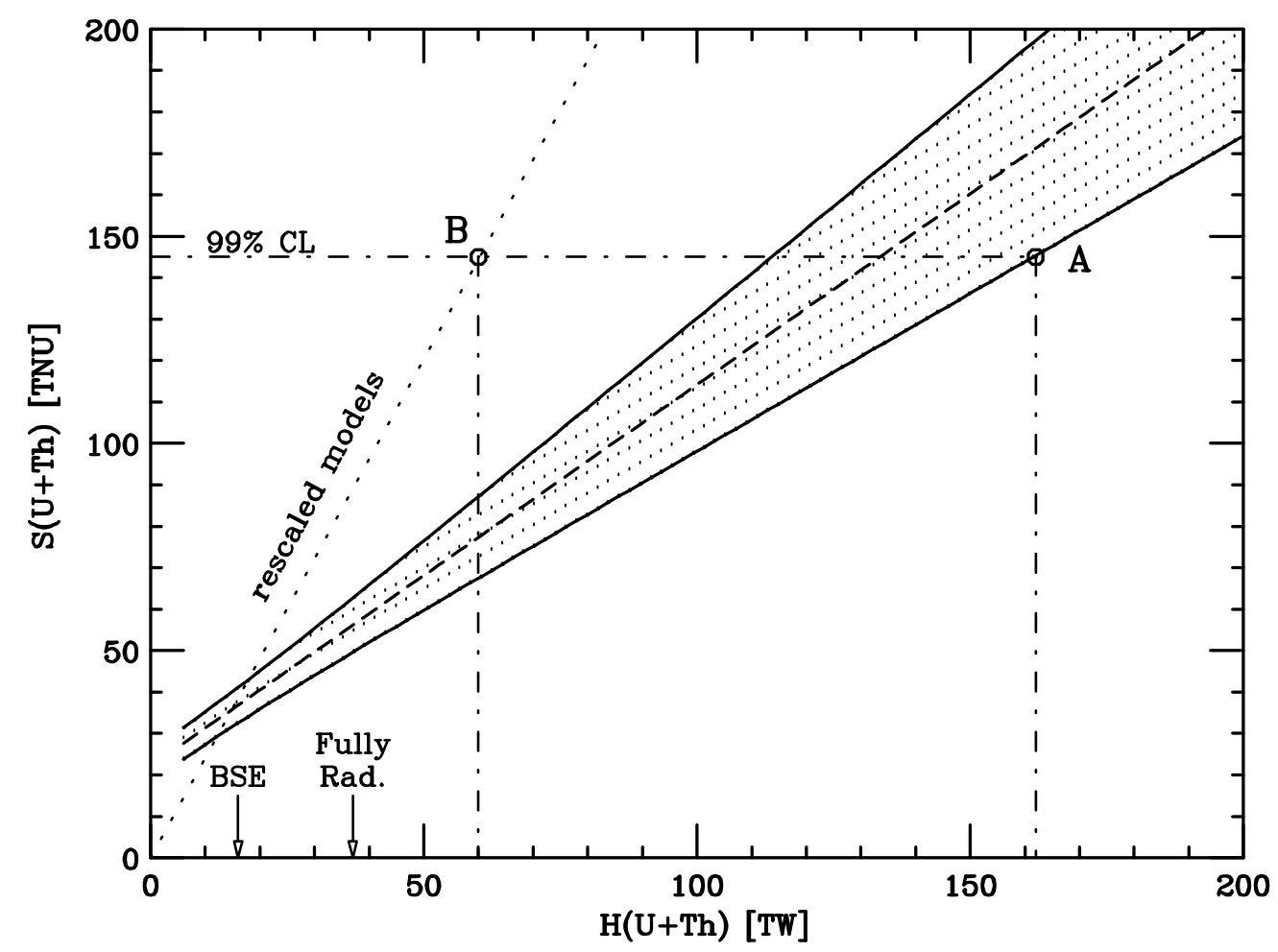

FIG. 1: The combined signal from Uranium and Thorium geo-neutrinos and the radiogenic heat production rate.

The shaded area denotes the region allowed by geochemical and geophysical constraints. The dashed median line represents our best estimate for the relationship between signal and power.

The dotted line denotes the "rescaled models" of Eq. [3, used in [10]. Note that most of these models are outside the allowed area.

One TNU corresponds to $10^{-32} \overline{\nu_{e}}$ captures per target proton per year. 


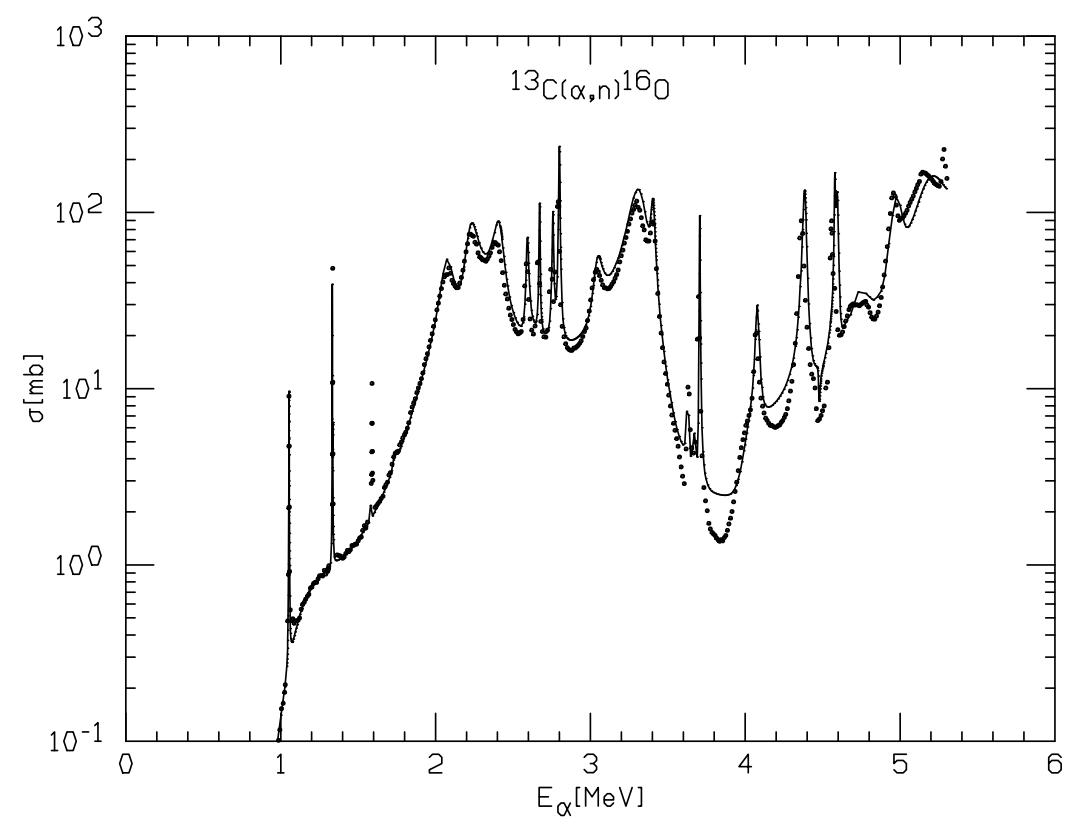

FIG. 2: Cross section of ${ }^{13} \mathrm{C}(\alpha, n){ }^{16} \mathrm{O}$. The solid line corresponds to the JENDL compilation, dots are the experimental points from Ref. [15]. 